While, in many cases, it may be expedient to carry out the integrations with respect to $\xi, \eta$ before integrating with respect to $\nu$, carrying out the latter integration yields ${ }^{5}$

$$
\phi(\infty, y, z)=\frac{z}{\pi} \int_{-\infty}^{\infty} d \xi \int_{-\infty}^{\infty} d \eta \frac{\gamma(\xi, \eta)}{z^{2}+(y-\eta)^{2}} .
$$

This result may also be obtained (or interpreted) through the method of source distributions. In the plane $z=0$, Eq. (7) reduces to

$$
\lim _{z \rightarrow 0+} \phi(\infty, y, z)=\int_{-\infty}^{\infty} d \xi \int_{-\infty}^{\infty} d \eta \gamma(\xi, \eta) \delta(y-\eta)=\int_{-\infty}^{\infty} d \xi \gamma(\xi, y) .
$$

It is of interest to remark that the results, Eqs. (6)-(8), are independent of Mach number and are, therefore, valid for all Mach numbers for which linearized theory is admissible. In view of Jones' results for low aspect ratio airfoils, ${ }^{6}$ this state of affairs is not unexpected.

'B. O. Peirce, A short table of integrals, Ginn and Co., New York, 1929, p. 64.

${ }^{6} \mathrm{R}$. T. Jones, Properties of low-aspect-ratio pointed wings at speeds below and above the speed of sound, NACA TN 1032, March, 1946.

\title{
A THEOREM CONCERNING THE POSITIONS OF EQUILIBRIUM OF A RIGID BODY*
}

\author{
By L. A. MACCOLL (Bell Telephone Laboratories)
}

The following interesting theorem is an immediate consequence of three theorems which are well known in certain mathematical circles. However, since, as far as the writer is aware, the theorem has not previously been stated explicitly, it seems worth while to present it in this note.

Theorem. Let $B$ be a rigid body, of any form, which is free to rotate about a fixed point $P$. Let $B$ be subjected to forces which are derived from a potential energy function $V$, which is an arbitrary single-valued function, of class $C^{2}$, of the coordinates of $B$. Then $B$ has at least four distinct positions of equilibrium.

We first note that the positions (or configurations) of equilibrium of $B$ are the points in the configuration space at which the function $V$, which is defined over the configuration space, is stationary. Then the theorem is an immediate consequence of the following three theorems.

1. The configuration space of a rigid body which is free to rotate about a fixed point is homeomorphic with real three-dimensional projective space.

2. The number of distinct stationary points of a real single-valued function, of class $C^{2}$, defined over a closed manifold $M$ is at least equal to a certain topological invariant of $M$, called the category of $M$.

3 . The category of real $n$-dimensional projective space is $n+1$.

The first of these theorems, in one or another of various equivalent forms, is widely known. In effect, it is given as an exercise on page 56 of Seifert and Threlfall's Lehrbuch der Topologie.

${ }^{*}$ Received Aug. 8, 1949. 
The second theorem is stated and proved on page 91 of Seifert and Threlfall's Variationsrechnung im Grossen.

The third theorem is stated and proved on pages 42 and 43 of Lusternik and Schnirelmann's monograph Méthodes Topologiques dans les problèmes variationnels, Première Partie, Paris, Hermann et $\mathrm{C}^{\mathrm{i}}$, 1934. (Actualités Scientifiques et Industrielles, No. 188.)

\section{NOTE ON THE KERNEL $\exp (-|x-y|)$ * \\ BY HARRY POLLARD (Cornell University)}

In a recent issue of this Quarterly a theorem was stated which, after some trivial changes and correction of a typographical error, reads as follows. ${ }^{1}$ In order that the function $f(x)$ have the form

$$
f(x)=\frac{1}{2} \int_{0}^{\infty} e^{-|x-y|} g(y) d y, \quad x \geq 0,
$$

it is necessary and sufficient that

$$
\begin{gathered}
f(0)=f^{\prime}(0) ; \\
g(x)=f(x)-f^{\prime \prime}(x) ; \\
g(x)=0\left(e^{d x}\right), x \rightarrow \infty, \text { for some } d<1 .
\end{gathered}
$$

Now the example $f(x)=e^{x}$ shows that the conditions are certainly not sufficient even if (c) is replaced by the condition

$$
\text { the integral in (1) exists, }
$$

which the author regards as equivalent to (c).

On the other hand the example $g(x)=e^{x}\left(x^{2}+1\right)^{-1}$ shows that the condition (c) is not necessary either. It also shows that (c) and $\left(c^{\prime}\right)$ are far from equivalent.

The following is a correct version of the theorem. The only difficulty is the discovery of a condition to replace (c). Once this is done the proof is straightforward, and is therefore omitted.

THEOREM. In order that $f(x)$ have the form (1) where $g(y)$ is a prescribed function integrable on each finite interval it is necessary and sufficient that $f^{\prime}(x)$ exists and is absolutely continuous on finite intervals, and moreover that

$$
\begin{gathered}
f(0)=f^{\prime}(0) ; \\
g(x)=f(x)-f^{\prime \prime}(x) \text { for almost all } x>0 ; \\
f^{\prime}(x)=o\left(e^{x}\right), x \rightarrow \infty .
\end{gathered}
$$

Remarks. (i) The condition (c) cannot be replaced by $O\left(e^{x}\right)$ as the example $f(x)=e^{x}$ shows. (ii) If the word "prescribed" and condition (b) are omitted simultaneously the theorem remains true. 